\title{
The Effect of an Upper Limb Rehabilitation Robot on Hemispatial Neglect in Stroke Patients
}

\author{
Yoon Sik Choi, MD, Kyeong Woo Lee, MD, Jong Hwa Lee, MD, \\ Sang Beom Kim, MD, Gyu Tae Park, MD, Sook Joung Lee, MD \\ Department of Physical Medicine and Rehabilitation, Dong-A University College of Medicine \& Busan-Ulsan Regional \\ Cardiocerebrovascular Center, Busan, Korea
}

\begin{abstract}
Objective To investigate the effectiveness of an upper limb rehabilitation robot therapy on hemispatial neglect in stroke patients.

Methods Patients were randomly divided into an upper limb rehabilitation robot treatment group (robot group) and a control group. The patients in the robot group received left upper limb training using an upper limb rehabilitation robot. The patients sat on the right side of the robot, so that the monitor of the robot was located on the patients' left side. In this position, patients could focus continuously on the left side. The control group received conventional neglect treatment, such as visual scanning training and range of motion exercises, administered by occupational therapists. Both groups received their respective therapies for 30 minutes a day, 5 days a week for 3 weeks. Several tests were used to evaluate treatment effects before and after the 3-week treatment.

Results In total, 38 patients (20 in the robot group and 18 in the control group) completed the study. After completion of the treatment sessions, both groups showed significant improvements in the Motor-Free Visual Perception Test 3rd edition (MVPT-3), the line bisection test, the star cancellation test, the Albert's test, the Catherine Bergego scale, the Mini-Mental State Examination and the Korean version of Modified Barthel Index. The changes in all measurements showed no significant differences between the two groups.

Conclusion This present study showed that the upper limb robot treatment had benefits for hemispatial neglect in stroke patients that were similar to conventional neglect treatment. The upper limb robot treatment could be a therapeutic option in the treatment of hemispatial neglect after stroke.
\end{abstract}

Keywords Stroke, Perceptual disorders, Robotics, Upper extremity

Received August 13, 2015; Accepted October 28, 2015

Corresponding author: Sook Joung Lee

Department of Physical Medicine and Rehabilitation, Dong-A University Medical Center, 26 Daesingongwon-ro, Seo-gu, Busan 49201, Korea. Tel: +8251-240-5690, Fax: +82-51-254-8511, E-mail: 1sj995414@hanmail.net

ORCID: Yoon Sik Choi (http://orcid.org/0000-0003-2266-541X); Kyeong Woo Lee (http://orcid.org/0000-0001-8785-5535); Jong Hwa Lee (http://orcid. org/0000-0003-2489-358X); Sang Beom Kim (http://orcid.org/0000-0002-5622-5933); Gyu Tae Park (http://orcid.org/0000-0002-7809-2561); Sook Joung Lee (http://orcid.org/0000-0002-6894-445X).

(c) This is an open-access article distributed under the terms of the Creative Commons Attribution Non-Commercial License (http://creativecommons.org/ licenses/by-nc/4.0) which permits unrestricted noncommercial use, distribution, and reproduction in any medium, provided the original work is properly cited. Copyright $\odot 2016$ by Korean Academy of Rehabilitation Medicine 


\section{INTRODUCTION}

Hemispatial neglect is a relatively frequent consequence of lesions from the right hemisphere stroke, and an important indicator of a poor functional outcome. It refers to the phenomenon of neglecting normal stimuli coming from the external space of the paretic side and showing no response to those stimuli, despite there being no problems with vision and the visual field [1]. Hemispatial neglect is also often expressed by other terms, such as visual neglect, visuospatial neglect, and visual inattention [2]. Hemispatial neglect frequently occurs when there is a lesion in the right inferior parietal lobe, thalamus, and basal ganglia [3-6]. As the right hemisphere plays a major role in spatial attention, the symptoms of hemispatial neglect are known to be more severe and persist longer than when a lesion is located in the right hemisphere compared to the left hemisphere [7-9]. In the case of hemispatial neglect patients, problems in the process of rehabilitation, such as a slow recovery from hemiplegia, balance problems, and increased hospitalization periods, are greater compared to stroke patients without hemispatial neglect [10-13].

Among hemispatial neglect treatment techniques, three of the most widely validated hemispatial neglect treatments are visual scanning training $[14,15]$, limb activation treatment [16], and prism adaptation treatment $[17,18]$. In addition, optokinetic stimulation, neck muscle vibration, trunk rotation, repetitive transcranial magnetic stimulation, eye patching, and music therapy are also used in treatment for hemispatial neglect [19].

Over the last decade, robots have been regarded as a potential tool to achieve muscle strength recovery in stroke patients through neurorehabilitation treatment, and the clinical utilization of robots has been increasing [20]. Besides, it has been found that upper limb rehabilitation methods using robot systems can provide safe and intensive treatment to subacute and chronic stroke patients [21]. In addition, there was an observational study showing improvements in arm function following robotassisted arm rehabilitation in stroke patients [22].

To our knowledge, no study has used upper limb rehabilitation robots in hemispatial neglect treatment, except for a case study. The aim of this study was to identify the effects and potential benefits of the upper limb rehabilitation robot on hemispatial neglect in subacute stroke patients. We hypothesized that if the upper limb rehabilitation robot treatment was carried out on the left side of the patient, the effects of visual scanning training, limb activation, or task-oriented treatment could be obtained.

\section{MATERIALS AND METHODS}

\section{Study subjects}

Right hemisphere stroke patients with left hemispatial neglect were recruited in this study from January 2014 to April 2015. Hemispatial neglect was diagnosed when bisection deviated $5 \mathrm{~mm}$ or more to the right side in the line bisection test [23]. Among the patients, those with any past medical history of brain damage, stroke, and other neurologic or neuropsychiatric disease were excluded from this study. Also, those who could not undergo robot treatment or hemispatial neglect tests due to severe cognitive impairment were excluded. Patients with below second-grade left upper extremity muscle strength in a manual muscle test, those who had any visual field defect, or those with a seriously declined sitting balance interfering with upper extremity rehabilitation robot treatment in a sitting position on a chair with a back and armrests, were also excluded from the study. Before enrollment in the study, all patients received general physical, occupational and cognitive therapies. Approval for the study was obtained from the Institutional Review Board of Dong-A University Hospital (No. 15-156), and written consent was received from all patients.

Included patients were randomly assigned either to the upper limb rehabilitation robot group (robot group) or to the control group. The patients in the robot group received hemispatial neglect treatment using the upper limb rehabilitation robot. The control group received conventional neglect treatment, such as visual scanning training and range of motion exercises, administered by occupational therapists. Both the upper limb rehabilitation robot and the control groups received treatment for 30 minutes per day, 5 days a week for 3 weeks. In addition, occupational therapists conducted conventional occupational therapy and daily living activity training on both groups for 30 minutes a day. Physical and cognitive therapies of the same length of time were applied to all patients. 


\section{Robot treatment programs}

The Neuro-X system (Apsun Inc., Seoul, Korea) was used for the robot treatment of hemispatial neglect. During the treatment, each patient sat on the right side of the robot so that the monitor was located to the left side of the patient (Fig. 1). In this position, the patient could focus continuously on the left side. The robot treatment program was implemented through games that induce passive and active assistive exercises of the wrist, elbow, and shoulder joints, and the games consisted of two types of isometric exercises and two types of range of motion exercises. The isometric exercises were comprised of an archery game which programmed to hit apples appearing on the left and right sides of the monitor without any determined order and a goalkeeper game, which programmed to block a ball randomly approaching the bottom left and right sides of the monitor (Fig. 2A, 2B). The range of motion exercises were conducted in a passive or an active assistive mode and they consisted of a dolphin circus game and a skateboard game (Fig. 2C, 2D). All the game programs prompted the participants' concentration through sound effects.

During robot treatment, occupational therapists monitored the patients for diligently following the progression of the robot programs, measured the patients' muscle strength before the robot programs began, and helped the patients when the games had to be changed from time to time.

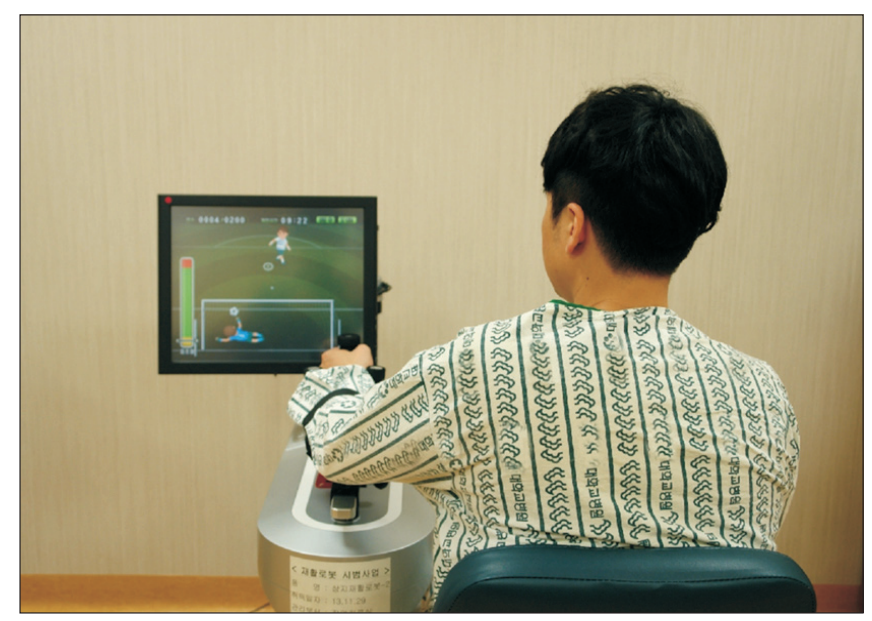

Fig. 1. Neuro-X system, an upper limb rehabilitation robot, consisted of a monitor and upper limb exercise equipment. The subject's posture was deviated at the right side from the robot.

\section{Evaluation methods}

Treatment effects were evaluated through the MotorFree Visual Perception Test 3rd edition (MVPT-3), the line bisection test, the star cancellation test, the Albert's test, the Catherine Bergego scale (CBS), the Mini-Mental State Examination (MMSE), and the Korean version of Modified Barthel Index (K-MBI). These indicators were measured before and after the 3-week treatment by the occupational therapists and physical therapists who did not participate firsthand in the treatment, and the CBS results were prepared with assistance from the caregivers.

The MVPT-3, made up of totaling 65 items, is a tool to evaluate the patient's visual perception and consists of six categories: visual discrimination, form consistency, visual short-term memory, visual closure, spatial orientation, and figure ground [24]. Higher scores indicate better visual perception abilities. In the line bisection test, differences between the centers of the lines perceived by the patient and the actual centers of the lines were divided by half of the bisected lines and the average value determined by adding all the values in percentages was taken as the resultant value of the line bisection test [25]. The star cancellation test evaluates the subject's scan ability and comprises 56 small stars, 52 large stars, words, and characters. In this test, the total number of small stars marked by the subject was evaluated. In the Albert's test, the number of lines marked by the patient out of the 18 lines located on the left side of the test paper was taken as the resultant value [26]. The CBS is the only tool that enables the evaluation of not only hemispatial neglect in personal (body parts or on the body surface), peripersonal (within an arm's reach), and extra-personal (beyond an arm's reach) spaces, but also perceptual and representational attention, and motor domains [27]. Moreover, the CBS enables direct evaluation of declines in ability to perform daily living activities and restrictions in participation using 10 items [19].

\section{Statistical analysis}

The SPSS ver. 21.0 for Windows (IBM, Armonk, NY, USA) was used for statistical analysis. Comparisons of the robot group and the control group were statistically processed through Mann-Whitney U tests. Also, an evaluation of all the indicators, including various indicators to determine the degree of hemispatial neglect, was con- 

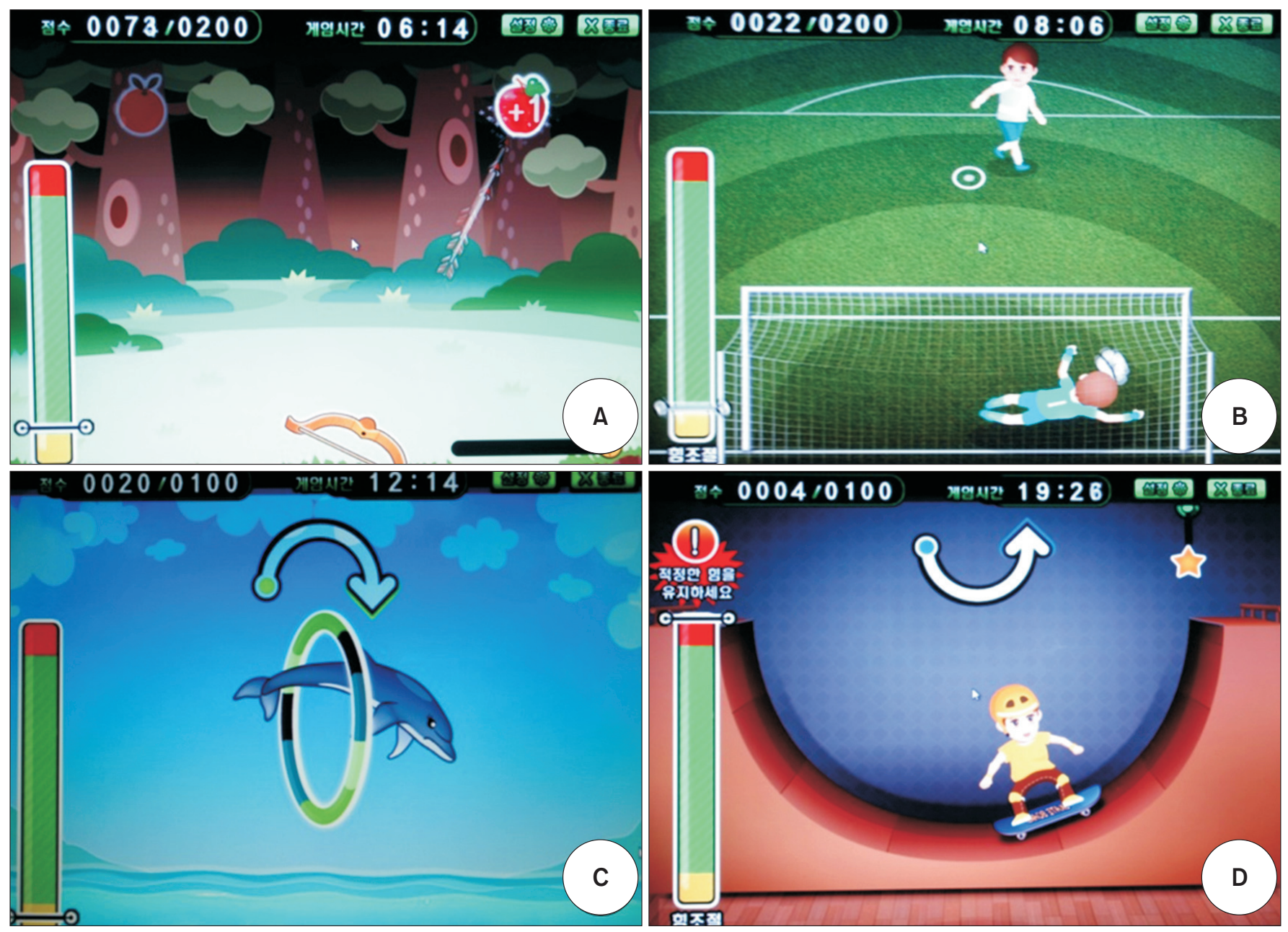

Fig. 2. Four game programs on the Neuro-X system (Apsun Inc., Seoul, Korea). (A) Archery game, (B) goalkeeper game, (C) dolphin circus game, and (D) skateboard game.

ducted using the Wilcoxon signed-rank test. Statistical significance was defined for cases where the p-value was below 0.05 .

\section{RESULTS}

During the study period, 124 right hemisphere stroke patients had been admitted to our department. In total, 78 patients were excluded from the study and 46 patients who had left hemispatial neglect after right hemisphere stroke were recruited. From these, 23 patients were assigned at random to either the robot group or the control group. Eight patients dropped out due to early discharge from the hospital or declines in their medical condition. Finally 38 patients, 20 in the robot group and 18 in the control group, completed all treatment sessions (Fig. 3).
The robot group consisted of 12 males and 8 females, and the mean age of the patients was 60.8 years. In the group, 13 patients had ischemic stroke, while 7 had hemorrhagic stroke, and the mean period after the onset of stroke was 37.4 days. The control group consisted of 8 males and 10 females, and the mean age of the patients was 63.2 years. Twelve of the patients had ischemic stroke, while 6 had hemorrhagic stroke, and the mean period that passed after the onset of stroke was 37.9 days. There was no significant difference between the two groups regarding the initial values of the evaluation factors, including baseline characteristics, the manual function test, and the Modified Ashworth Scale (Table 1). After the 3-week hemispatial neglect treatment, significant improvements were observed in both groups compared to before the treatment in the values of the MVPT, the line bisection, 


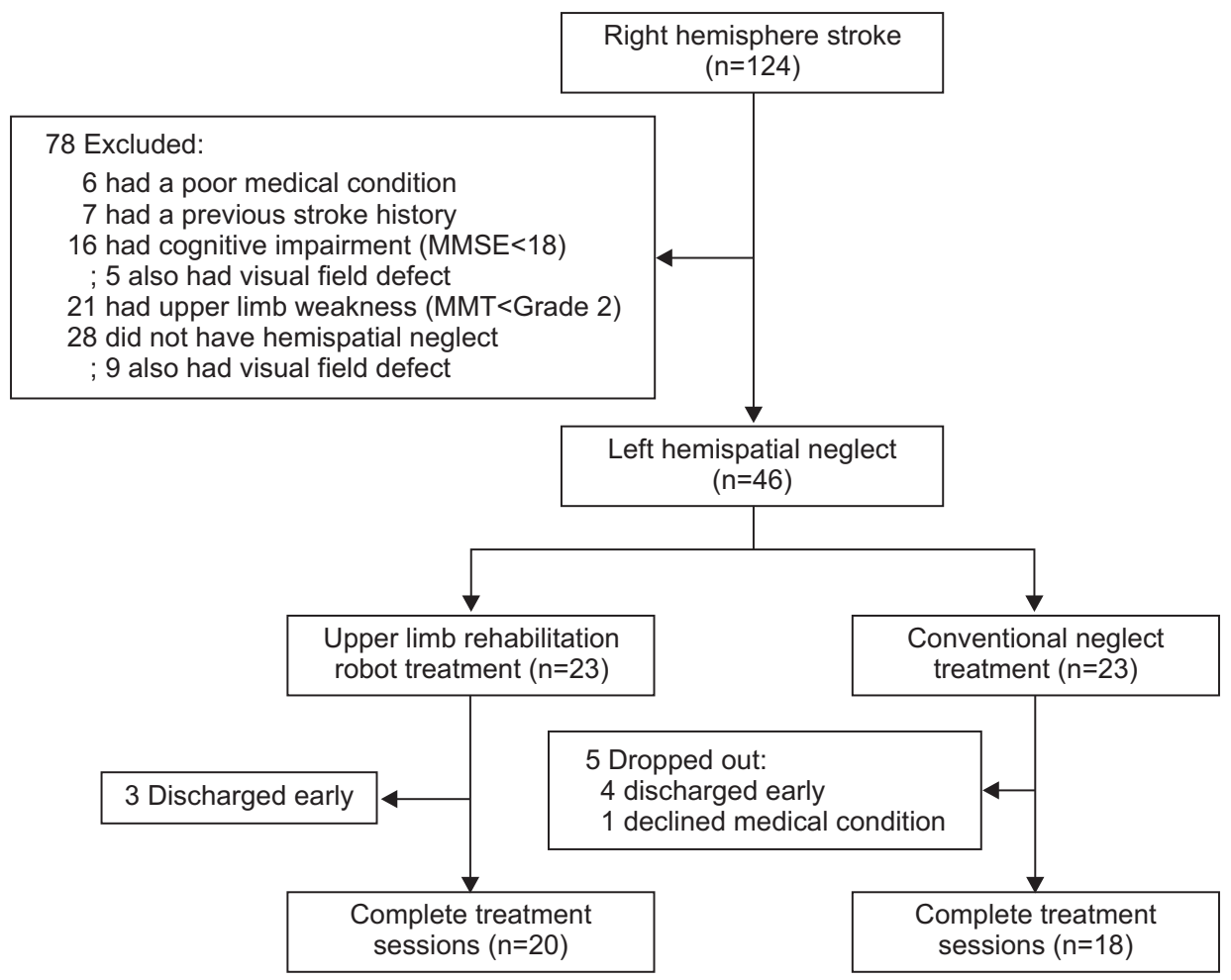

Fig. 3. The algorithm for enrollment of the subjects. MMSE, Mini-Mental State Examination; MMT, Manual Muscle Test.

Table 1. Baseline characteristics of two groups

\begin{tabular}{|lccc|}
\hline \multicolumn{1}{c}{ Variable } & RG $(\mathbf{n = 2 0})$ & CG (n=18) & p-value \\
\hline Age (yr) & $60.75 \pm 12.54$ & $63.17 \pm 11.12$ & 0.482 \\
\hline Sex (male:female) & $12: 8$ & $8: 10$ & \\
\hline Lesion (ischemic:hemorrhagic) & $13: 7$ & $12: 6$ & 0.977 \\
Duration (day) & $37.40 \pm 15.93$ & $37.94 \pm 16.93$ & 0.536 \\
\hline MFT & $5.90 \pm 3.63$ & $5.22 \pm 3.20$ & 0.508 \\
\hline MAS & $0.35 \pm 0.56$ & $0.50 \pm 0.69$ & 0.649 \\
\hline MVPT-3 & $17.70 \pm 6.33$ & $16.11 \pm 4.17$ & 0.930 \\
\hline Line bisection & $36.62 \pm 14.24$ & $35.93 \pm 13.28$ & 0.387 \\
Star cancellation & $17.80 \pm 10.31$ & $13.83 \pm 7.73$ & 0.217 \\
Albert's test & $7.20 \pm 4.40$ & $5.44 \pm 3.24$ & 0.340 \\
CBS & $22.50 \pm 4.14$ & $23.89 \pm 3.14$ & 0.676 \\
\hline MMSE & $20.95 \pm 3.19$ & $20.44 \pm 2.68$ & 0.341 \\
\hline K-MBI & $24.30 \pm 9.98$ & $21.06 \pm 10.76$ & \\
\hline
\end{tabular}

Values are presented as mean \pm standard deviation.

RG, robot group; CG, control group; MFT, Manual Function Test; MAS, Modified Ashworth Scale; MVPT-3, Motor-Free Visual Perception Test-3rd edition; CBS, Catherine Bergego Scale; MMSE, Mini-Mental State Examination; K-MBI, Korean version of Modified Barthel Index.

${ }^{*} \mathrm{p}<0.05$ by Mann-Whitney U test.

the star cancellation test, the Albert's test, the CBS, the MMSE, and the K-MBI $(\mathrm{p}<0.05)$ (Table 2). No significant differences were observed in the extent of change before and after treatment for any of the individual indicators between the two groups (Table 3). During the robot treatment, there were no treatment side effects, such as dizziness and upper extremity pain. 
Table 2. Change of measurements by neglect treatment

\begin{tabular}{|c|c|c|c|c|}
\hline \multirow{2}{*}{ Measurement } & \multicolumn{2}{|c|}{ RG $(n=20)$} & \multicolumn{2}{|c|}{ CG $(n=18)$} \\
\hline & Pre & Post & Pre & Post \\
\hline MVPT-3 & $17.70 \pm 6.33$ & $25.90 \pm 9.21^{*}$ & $16.11 \pm 4.17$ & $22.89 \pm 7.77^{*}$ \\
\hline Line bisection & $36.62 \pm 14.24$ & $25.53 \pm 11.32^{*}$ & $35.93 \pm 13.28$ & $28.46 \pm 12.56^{*}$ \\
\hline Star cancellation & $17.80 \pm 10.31$ & $25.60 \pm 11.51^{*}$ & $13.83 \pm 7.73$ & $19.78 \pm 8.93^{*}$ \\
\hline Albert's test & $7.20 \pm 4.40$ & $11.05 \pm 3.90^{*}$ & $5.44 \pm 3.24$ & $9.89 \pm 4.24^{*}$ \\
\hline CBS & $22.50 \pm 4.14$ & $18.80 \pm 4.94^{*}$ & $23.89 \pm 3.14$ & $19.61 \pm 3.53^{*}$ \\
\hline MMSE & $20.95 \pm 3.19$ & $22.40 \pm 3.14^{*}$ & $20.44 \pm 2.68$ & $21.67 \pm 2.50^{*}$ \\
\hline K-MBI & $24.30 \pm 9.98$ & $36.85 \pm 11.98^{*}$ & $21.06 \pm 10.76$ & $33.78 \pm 12.44^{*}$ \\
\hline
\end{tabular}

Values are presented as mean \pm standard deviation.

RG, robot group; CG, control group; MVPT-3, Motor-Free Visual Perception Test-3rd edition; CBS, Catherine Bergego Scale; MMSE, Mini-Mental State Examination; K-MBI, Korean version of Modified Barthel Index.

${ }^{*} \mathrm{p}<0.05$ by Wilcoxon signed-rank test.

Table 3. Changes of measurements between two groups

\begin{tabular}{lrcc}
\hline & RG (n=20) & CG (n=18) & p-value \\
\hline$\Delta$ MVPT-3 & $8.20 \pm 4.85$ & $6.56 \pm 4.87$ & 0.411 \\
$\Delta$ Line bisection & $-11.10 \pm 9.40$ & $-7.46 \pm 10.11$ & 0.306 \\
$\Delta$ Star cancellation & $7.90 \pm 5.46$ & $5.94 \pm 4.96$ & 0.325 \\
$\Delta$ Albert's test & $3.85 \pm 2.08$ & $4.44 \pm 2.66$ & 0.477 \\
$\Delta$ CBS & $-3.70 \pm 2.25$ & $-4.28 \pm 1.53$ & 0.415 \\
$\Delta$ MMSE & $1.45 \pm 2.06$ & $1.22 \pm 2.13$ & 0.656 \\
$\Delta$ K-MBI & $12.55 \pm 4.12$ & $12.72 \pm 4.00$ & 0.735 \\
\hline
\end{tabular}

Values are presented as mean \pm standard deviation.

RG, robot group; CG, control group; MVPT-3, MotorFree Visual Perception Test-3rd edition; CBS, Catherine Bergego Scale; MMSE, Mini-Mental State Examination; K-MBI, Korean version of Modified Barthel Index. ${ }^{*} \mathrm{p}<0.05$ by Mann-Whitney $\mathrm{U}$ test.

\section{DISCUSSION}

The present study aimed to identify the effects of an upper limb rehabilitation robot on hemispatial neglect. Our results showed that the upper limb rehabilitation robot treatment had therapeutic effects similar to those of the conventional hemispatial neglect treatment. We also demonstrated an improvement in upper extremity function by using the upper limb rehabilitation robot that had already been proven.

We hypothesized that our methods of applying the upper limb rehabilitation robot to the left side of the patients could have similar effects to those of the conventional neglect treatment, such as visual scanning training, limb activation, or task-oriented treatment.
Visual scanning training was to lead to a re-orientation of visual scanning toward the neglected side. It is the most common and the best-studied neglect rehabilitative intervention, because of its ease of administration and cost-effectiveness. Many studies have compared a specific neglect therapy to visual scanning training, the effectiveness of which has been proven [2]. In the present study, the reason the upper limb rehabilitation robots showed effects similar to those of visual scanning training-centered conventional hemispatial neglect treatment is that the game programs appearing on the monitor located on the left played the role of visual scanning training.

The motor circuits and the attention functions of the brain are closely linked, as the activation of the upper limb on the opposite side of the lesion stimulates neural networks in the brain that assist in space perception [28]. Several studies have reported that movements of the left arm toward the left side space relieve hemispatial neglect $[29,30]$. In addition, Brunila et al. [31] published study results indicating that visual scanning training and limb activating treatment conducted together can serve to enhance the generalization of the effect to functional skills. In our study, upper limb rehabilitation could activate the left upper arm toward the left side space by task-oriented actions consisting of active and passive movements.

The rehabilitation robot treatment, has received attention recently for stroke rehabilitation, as it enables stroke patients to perform repetitive and intensive task-specific movements with the appropriate help and guidance of therapists [32]. During the upper limb rehabilitation ro- 
bot treatment, information on the monitor can provide visual feedback to help goal-oriented movements [20]. In addition, robot games can arouse and maintain the patients' interest in treatment.

According to the literature review, multiple previous studies have revealed the effect of the upper limb rehabilitation robot on functional improvement in stroke patients. In a large-scale upper limb rehabilitation robot study conducted with chronic stroke patients with moderate to severe functional impairment, Lo et al. [33] reported on results that proved the long-term effects of intensive upper limb rehabilitation robot treatment. Mazzoleni et al. [21] applied upper limb rehabilitation robots that could horizontally move to 25 sub-acute and chronic stroke patients and they reported that upper limb rehabilitation robots were effective for improvements in functional disorders of the upper extremities after stroke.

However, hemispatial neglect is a disease resulting from many heterogeneous disorders, and as the degrees of neurological deficits and the subtypes of hemispatial neglect differ by the patient, it is difficult to select the most effective option from many hemispatial neglect treatments [1]. Recently, a case study reported that the extent of hemispatial neglect decreased after passive joint movements of the left hand using a hand rehabilitation robot for two weeks in patients with left hemispatial neglect [34]. Although this case series study was the first study to use rehabilitation robots in the treatment of hemispatial neglect, it was limited in that there was no control group and only passive movements of the hand were performed. By comparison, our study is considered meaningful in that upper limb rehabilitation robots that enable passive and active movements of the upper extremities were compared with conventional hemispatial neglect treatments for the first time.

The present study had several limitations. First, the number of patients was small and the long-term effects of upper limb rehabilitation robots for hemispatial neglect treatment were not evaluated. Second, a sham group of patients who did not receive hemispatial neglect treatment was not included in the study due to ethical reasons. Thus the possibility that improvements in hemispatial neglect were natural could not be eliminated. Third, as hemispatial neglect evaluation tools, except for the CBS, were limited to the evaluation of hemispatial neglect in the peri-personal space, selection bias could not be fully eliminated. Larger studies with more patients and with long-term follow-up, as well as evaluation indicators that can evaluate subtypes of hemispatial neglect, are considered necessary.

In conclusion, the present study showed that upper limb robot treatment had benefits for hemispatial neglect in stroke patients that was similar to conventional neglect treatment. Applying the upper limb rehabilitation robot treatment on the left side of patients could induce the effects of visual scanning, limb activation, or task-oriented treatment. Our method was also safe and easy to apply in clinical settings. We suggest that this upper limb robot treatment could be a therapeutic option in the treatment of hemispatial neglect after stroke.

\section{CONFLICT OF INTEREST}

No potential conflict of interest relevant to this article was reported.

\section{ACKNOWLEGDMENTS}

This study was promoted as part of the spread business of market creation type robots of the Ministry of Trade, Industry and Energy and was carried out by rehabilitation robots supplied from the Ministry of Health \& Welfare and the National Rehabilitation Center in 2013.

\section{REFERENCES}

1. Luaute J, Halligan P, Rode G, Rossetti Y, Boisson D. Visuo-spatial neglect: a systematic review of current interventions and their effectiveness. Neurosci Biobehav Rev 2006;30:961-82.

2. Ting DS, Pollock A, Dutton GN, Doubal FN, Ting DS, Thompson M, et al. Visual neglect following stroke: current concepts and future focus. Surv Ophthalmol 2011;56:114-34.

3. Pierce SR, Buxbaum LJ. Treatments of unilateral neglect: a review. Arch Phys Med Rehabil 2002;83:25668.

4. Heilman KM, Valenstein E. Frontal lobe neglect in man. Neurology 1972;22:660-4.

5. Watson RT, Heilman KM. Thalamic neglect. Neurology 1979;29:690-4.

6. Damasio AR, Damasio H, Chui HC. Neglect following 
damage to frontal lobe or basal ganglia. Neuropsychologia 1980;18:123-32.

7. Halligan PW, Marshall JC. Toward a principled explanation of unilateral neglect. Cogn Neuropsychol 1994;11:167-206.

8. Posner MI, Petersen SE. The attention system of the human brain. Annu Rev Neurosci 1990;13:25-42.

9. Bailey MJ, Riddoch MJ, Crome P. Treatment of visual neglect in elderly patients with stroke: a single-subject series using either a scanning and cueing strategy or a left-limb activation strategy. Phys Ther 2002;82:78297.

10. Paolucci S, Antonucci G, Guariglia C, Magnotti L, Pizzamiglio L, Zoccolotti P. Facilitatory effect of neglect rehabilitation on the recovery of left hemiplegic stroke patients: a cross-over study. J Neurol 1996;243:308-14.

11. Paolucci S, Traballesi M, Gialloreti LE, Pratesi L, Lubich S, Antonucci G, et al. Changes in functional outcome in inpatient stroke rehabilitation resulting from new health policy regulations in Italy. Eur J Neurol 1998;5:17-22.

12. Rode G, Tiliket C, Boisson D. Predominance of postural imbalance in left hemiparetic patients. Scand J Rehabil Med 1997;29:11-6.

13. Kerkhoff G. Spatial hemineglect in humans. Prog Neurobiol 2001;63:1-27.

14. Weinberg J, Diller L, Gordon WA, Gerstman LJ, Lieberman A, Lakin $\mathrm{P}$, et al. Visual scanning training effect on reading-related tasks in acquired right brain damage. Arch Phys Med Rehabil 1977;58:479-86.

15. Antonucci G, Guariglia C, Judica A, Magnotti L, Paolucci S, Pizzamiglio L, et al. Effectiveness of neglect rehabilitation in a randomized group study. J Clin Exp Neuropsychol 1995;17:383-9.

16. Robertson IH, North N. Spatio-motor cueing in unilateral left neglect: the role of hemispace, hand and motor activation. Neuropsychologia 1992;30:553-63.

17. Rossetti Y, Rode G, Pisella L, Farne A, Li L, Boisson D, Perenin MT. Prism adaptation to a rightward optical deviation rehabilitates left hemispatial neglect. Nature 1998;395:166-9.

18. Frassinetti F, Angeli V, Meneghello F, Avanzi S, Ladavas E. Long-lasting amelioration of visuospatial neglect by prism adaptation. Brain 2002;125(Pt 3):60823.

19. Priftis K, Passarini L, Pilosio C, Meneghello F, Pitteri
M. Visual scanning training, limb activation treatment, and prism adaptation for rehabilitating left neglect: who is the winner? Front Hum Neurosci 2013;7:360.

20. Lo AC. Clinical designs of recent robot rehabilitation trials. Am J Phys Med Rehabil 2012;91(11 Suppl 3):S204-16.

21. Mazzoleni S, Sale P, Tiboni M, Franceschini M, Carrozza MC, Posteraro F. Upper limb robot-assisted therapy in chronic and subacute stroke patients: a kinematic analysis. Am J Phys Med Rehabil 2013;92(10 Suppl 2):e26-37.

22. Kim J, Park SW, Lee Y, Seo H. Clinical outcomes of robot-assisted arm rehabilitation in stroke patients. Brain Neurorehabil 2015;8:46-52.

23. Kerkhoff G, Bucher L, Brasse M, Leonhart E, Holzgraefe M, Volzke V, et al. Smooth pursuit "bedside" training reduces disability and unawareness during the activities of daily living in neglect: a randomized controlled trial. Neurorehabil Neural Repair 2014;28:554-63.

24. Mercier L, Desrosiers J, Hebert R, Rochette A, Dubois MF. Normative data for the motor-free visual perception test-vertical. Phys Occup Ther Geriatr 2001;19:3950.

25. Schenkenberg T, Bradford DC, Ajax ET. Line bisection and unilateral visual neglect in patients with neurologic impairment. Neurology 1980;30:509-17.

26. Albert ML. A simple test of visual neglect. Neurology 1973;23:658-64.

27. Chen P, Hreha K, Fortis P, Goedert KM, Barrett AM. Functional assessment of spatial neglect: a review of the Catherine Bergego scale and an introduction of the Kessler foundation neglect assessment process. Top Stroke Rehabil 2012;19:423-35.

28. Rizzolatti G, Berti A. Neglect as a neural representation deficit. Rev Neurol (Paris) 1990;146:626-34.

29. Robertson IH, North NT, Geggie C. Spatiomotor cueing in unilateral left neglect: three case studies of its therapeutic effects. J Neurol Neurosurg Psychiatry 1992;55:799-805.

30. Gainotti G, Perri R, Cappa A. Left hand movements and right hemisphere activation in unilateral spatial neglect: a test of the interhemispheric imbalance hypothesis. Neuropsychologia 2002;40:1350-5.

31. Brunila T, Lincoln N, Lindell A, Tenovuo O, Ham- 
alainen $\mathrm{H}$. Experiences of combined visual training and arm activation in the rehabilitation of unilateral visual neglect: a clinical study. Neuropsychol Rehabil 2002;12:27-40.

32. Masiero S, Carraro E, Ferraro C, Gallina P, Rossi A, Rosati G. Upper limb rehabilitation robotics after stroke: a perspective from the University of Padua, Italy. J Rehabil Med 2009;41:981-5.

33. Lo AC, Guarino PD, Richards LG, Haselkorn JK, Wit- tenberg GF, Federman DG, et al. Robot-assisted therapy for long-term upper-limb impairment after stroke. N Engl J Med 2010;362:1772-83.

34. Varalta V, Picelli A, Fonte C, Montemezzi G, La Marchina E, Smania N. Effects of contralesional robot-assisted hand training in patients with unilateral spatial neglect following stroke: a case series study. J Neuroeng Rehabil 2014;11:160. 